\title{
Fibroblast Growth Factor 23 Trajectories in Chronic Hemodialysis Patients: Lessons from the HEMO Study
}

\author{
Anna Jovanovich ${ }^{\mathrm{a}}$ b Zhiying You $^{\mathrm{b}}$ Tamara Isakova $^{\mathrm{c}}$ Kristen Nowak $^{\mathrm{b}}$ \\ Alfred Cheung $^{\mathrm{d}, \mathrm{e}}$ Myles Wolf ${ }^{f}$ Michel Chonchol $^{\mathrm{b}}$ Jessica Kendrick ${ }^{\mathrm{b}}$ \\ a Renal Section, Medical Service, Veteran Affairs Eastern Colorado Health Care System, Denver, CO, USA; ${ }^{b}$ Division \\ of Renal Diseases and Hypertension, Department of Medicine, University of Colorado Denver Anschutz Medical \\ Campus, Aurora, CO, USA; ' Division of Nephrology and Hypertension, Department of Medicine and Center for \\ Translational Metabolism and Health, Institute for Public Health and Medicine, Northwestern University Feinberg \\ School of Medicine, Chicago, IL, USA; d Division of Nephrology and Hypertension, Department of Medicine,

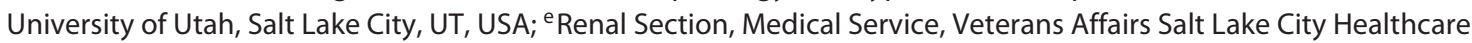

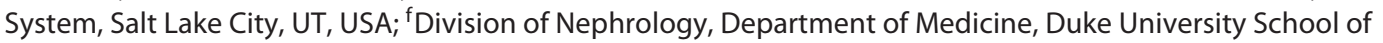 \\ Medicine, Durham, NC, USA
}

\section{Keywords}

Fibroblast growth factor $23 \cdot$ Hemodialysis · Trajectories

\begin{abstract}
Background: Long-term patterns of fibroblast growth factor 23 (FGF23) are poorly characterized among dialysis patients. Objectives: To identify different FGF23 trajectories and determine clinical factors that predict distinct FGF23 trajectories and whether FGF23 trajectories differ in regard to their associations with all-cause mortality among prevalent hemodialysis patients. Methods: The HEMO study was a randomized multicenter study evaluating the effects of highdose vs. standard-dose and high-flux vs. low-flux hemodialysis on mortality. We measured intact FGF23 levels in stored serum samples at baseline and annually among $919 \mathrm{HEMO}$ participants and identified FGF23 trajectories using groupbased modeling. Logistic regression determined predictors of trajectories. Cox regression models evaluated the association between trajectory and all-cause mortality. Results: We
\end{abstract}

identified 5 distinct FGF23 trajectory groups during the initial 24 months: low stable, low increasing, elevated increasing, elevated decreasing, and elevated stable. In multivariable models, diabetes, high dose dialysis, no venous catheter, low serum calcium, phosphorus, and interleukin-6, no vitamin $\mathrm{D}$ analog use, and greater residual kidney function were associated with the low stable trajectory group compared to the elevated stable group. High flux dialysis, no venous catheter, and low serum phosphorus and 25-hydroxyvitamin D were associated with the elevated decreasing trajectory group compared to the elevated stable group. After full adjustment, the low stable trajectory group was associated with reduced mortality (hazard ratio [HR] $0.61 ; 95 \% \mathrm{Cl}$ 0.41-0.91) compared to the elevated stable trajectory group. Conclusions: We identified 5 distinct FGF23 trajectories over 24 months among HEMO study participants including a decreasing trajectory. The low stable FGF23 trajectory was associated with a reduced $\mathrm{HR}$ of all-cause mortality.

(c) 2019 S. Karger AG, Basel

\section{KARGER}

(C) 2019 S. Karger AG, Basel

E-Mail karger@karger.com

www.karger.com/ajn
Anna Jovanovich

Division of Renal Diseases and Hypertension, Department of Medicine

University of Colorado Denver Anschutz Medical Campus

13199 East Montview Blvd., Suite 495, Aurora, CO 80045 (USA)

E-Mail anna.jovanovich@ucdenver.edu 


\section{Introduction}

Patients with end-stage kidney disease (ESKD) have a high mortality rate and the leading cause of death is cardiovascular disease (CVD), which accounts for more than half of all deaths [1]. Abnormal mineral metabolism is a hallmark of ESKD and is characterized by hyperphosphatemia, secondary hyperparathyroidism, and fibroblast growth factor 23 (FGF23) excess [2]. FGF23 is a hormone that regulates phosphorus and 1,25-dihydroxyvitamin $\mathrm{D}\left(1,25[\mathrm{OH}]_{2} \mathrm{D}\right)$, directly induces left ventricular hypertrophy in animal models [3], and is associated with anemia [4], infection [5], and left ventricular hypertrophy [6] in ESKD cohort studies. Large prospective investigations including a spectrum of chronic kidney disease (CKD) patients have shown that baseline or time-averaged FGF23 predicts CVD and all-cause mortality risk [5, 7-11]. An analysis of Chronic Renal Insufficiency Cohort (CRIC) participants demonstrated 3 distinct trajectories of FGF23 levels. Participants whose FGF23 trajectory increased over 5 years demonstrated a 4-15-fold increased risk of mortality compared to the stable FGF23 trajectory [12].

No prior studies have described the full spectrum of different FGF23 trajectories among prevalent hemodialysis patients. We used trajectory modeling to describe FGF23 trajectories over 24 months among HEMO study [13] participants. The HEMO study was designed to examine whether greater dialytic urea removal or high-flux dialysis improves clinical outcomes in prevalent hemodialysis patients. We conducted this post hoc analysis of the HEMO study to test the hypotheses that (1) different FGF23 trajectories exist over time; (2) clinical factors predict distinct FGF23 trajectories; and (3) low stable FGF23 trajectories are associated with decreased risk of all-cause mortality among prevalent hemodialysis patients.

\section{Materials and Methods}

\section{Study Population}

The HEMO study was a prospective, randomized, multicenter clinical trial with a $2 \times 2$ factorial design [13]. A total of 1,846 hemodialysis patients were randomly assigned to either low-flux or high-flux membrane dialyzers and to either standard dose dialysis targeting an equilibrated dose ( $\mathrm{eKt} / \mathrm{V}$ of urea) of 1.05 or a high dose targeting an eKt/V of urea of 1.45 . Among the eligibility criteria were (1) a minimum of 3 months on hemodialysis and (2) residual kidney urea clearance of $<1.5 \mathrm{~mL} / \mathrm{min}$ per $35 \mathrm{~L}$ of urea distribution volume to limit the contribution from native kidneys and maximize the relative effect of dialysis on total body solute clearance. The pri- mary outcome, all-cause mortality, did not differ among treatment arms. FGF23 was previously measured in a total of 1,441 HEMO participants [5]. This post hoc analysis included alive participants with at least 2 (up to 3 ) yearly FGF23 measurements in the initial 24 months of the HEMO study $(n=919)$. Of the 522 HEMO participants with FGF23 measurements not included in this analysis, 204 (39\%) died within the first 24 months of enrollment. All participants provided written informed consent, and the study had institutional review board approval at participating centers.

\section{Exposure Variables}

The primary exposure variables were FGF23 trajectories derived from 2 to 3 serum FGF23 measurements at baseline, 12- and 24 -months. All serum samples were drawn prior to dialysis treatment and stored in a central repository at $-80^{\circ} \mathrm{C}$ until they were shipped to the University of Washington for analysis. The Kainos immunoassay, which detects the full-length, biologically intact FGF23 molecule via midmolecule and distal epitopes, measured intact FGF23. We measured FGF23 in duplicate and used the blinded replicate samples to calculate intra and inter-assay coefficients of variations, which were 3.8 and $3.0 \%$, respectively. FGF23 stability in frozen samples is previously described [14].

\section{Outcomes}

The outcome was time to all-cause mortality, the HEMO study primary outcome. Outcomes were classified at clinical centers and reviewed by a committee of HEMO study investigators unaware of treatment assignments [13]. Follow-up for mortality in this subcohort began at month 24, when the final FGF23 measurement and trajectory group membership was ascertained. Median (interquartile range) follow-up was 36 (23-36) months and reflects death and transplant.

\section{Covariables}

All covariables were ascertained at the baseline visit of the original HEMO study and included: age, sex, race, dialysis duration, diabetic status (past or current use of hypoglycemic agents), comorbid medical conditions, cigarette smoking history, central venous catheter as hemodialysis vascular access, and vitamin D analog use. Serum albumin, calcium, phosphorus and intact parathyroid hormone (PTH) level were measured at baseline. Inflammatory markers, high-sensitivity C-reactive protein, interleukin-6 (IL-6), tumor necrosis factor- $\alpha$, and interferon- $\gamma$, and total 25 -hydroxyvitamin $\mathrm{D}$ $\left(25[\mathrm{OH}] \mathrm{D}\right.$; sum of $25[\mathrm{OH}] \mathrm{D}_{2}$ and $\left.25[\mathrm{OH}] \mathrm{D}_{3}\right)$ and $1,25(\mathrm{OH})_{2} \mathrm{D} \mathrm{lev-}$ els were measured in stored baseline serum samples. Online supplementary methods (for all online suppl. material, see www.karger. com/doi/10.1159/000497445) for details. Urea clearance (mL/min) per $35 \mathrm{~L}$ of urea distribution volume determined residual kidney function [13].

\section{Statistical Analyses}

The chi-square test and analysis of variance compared baseline covariables across FGF23 trajectories. We log transformed skewed variables. We used group-based trajectory modeling to identify distinct FGF23 patterns using 2-3 measures from the initial 24 months of the HEMO study. The SAS procedure, PROC TRAJ, fits a semi-parametric (discrete) mixture model to longitudinal data with the use of the maximum-likelihood method, using a censored normal model [15-17]. The Bayesian information criterion evaluated model fit $[15,18]$. We determined 5 
trajectory groups based on Bayesian information criterion, sample size considerations, and trajectory patterns. Participants were assigned to the trajectory group for which they had the maximum estimated probability of assignment. The mean probability of the final group membership was 0.75 (ranging 0.66-0.85) across trajectories. While there is no consensus, an average mean probability of 0.7 has been proposed as an adequate threshold [16].

Multinomial logistic regression identified baseline covariables (described above) with FGF23 trajectory group membership. To identify covariables for the multinomial logistic regression model, we used criteria based on the HEMO study design, subject-matter considerations, and biological understanding.

Cox regression models, adjusted for 5 nested sets of covariables, examined the association of trajectory group membership with all-cause mortality after month 24 of the HEMO study. Model 1 adjusted for demographics including age, sex, and race. Model 2 added comorbidities and dialysis-related factors: diabetic status, history of cardiac disease, number of years on dialysis, dose and flux treatment assignments in the HEMO study, baseline use of central catheter as vascular access, and baseline serum albumin. Model 3 added baseline serum calcium, phosphorus, iPTH, $25(\mathrm{OH}) \mathrm{D}$, and $1,25(\mathrm{OH})_{2} \mathrm{D}$ and circulating inflammatory markers (high-sensitivity C-reactive protein, IL-6, tumor necrosis factor- $\alpha$, and interferon $-\gamma$ ) because mineral metabolism and inflammation are potential confounders on the causal pathway between the exposure variable and outcome. Models 4 and 5 added baseline vitamin $\mathrm{D}$ analog use and residual kidney function, respectively, because these covariables may directly affect circulating FGF23 levels.

Proportional hazards assumption was evaluated using plots of Schoenfeld residuals and consideration of interaction terms between predictor variables and follow-up time. We considered models with cubic splines in quantitative variables and plots of martingale residuals to identify deviations from the linearity assumptions of the Cox regression models. Multiple imputation handled missing covariables. Two-tailed values of $p<0.05$ were considered statistically significant without formal adjustment for multiple comparisons. All statistical analyses were performed with SAS software, version 9.4 (SAS Institute, Cary, NC, USA).

\section{Results}

We identified 5 distinct FGF23 trajectory groups among 919 HEMO participants using 2-3 serum FGF23 measurements (58\% had 2 and $42 \%$ had 3 FGF23 measures) obtained at baseline, 12 and 24 months (Fig. 1): low stable, low increasing, elevated increasing, elevated decreasing, and elevated stable. In contrast to the 3 stable or increasing FGF23 trajectories reported in predialysis CKD patients [12], we found a distinct FGF23 trajectory that was elevated at baseline but decreased over time. The elevated increasing and the elevated decreasing trajectories had similar median baseline FGF23 values $(2,981$ and $3,541 \mathrm{pg} / \mathrm{mL}$, respectively) but

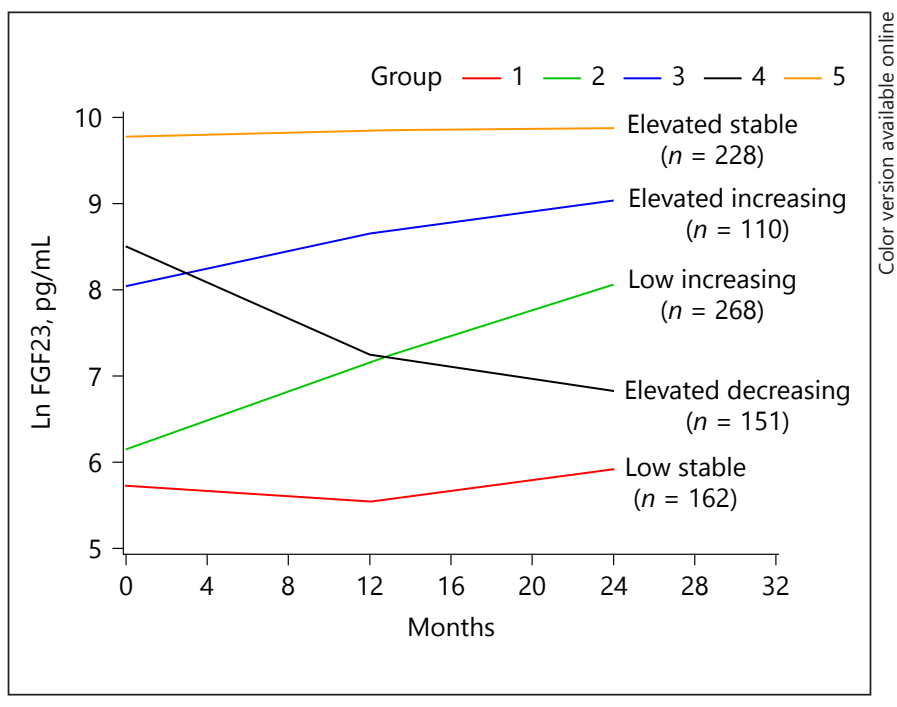

Fig. 1. FGF23 trajectories over 24 months. FGF23, fibroblast growth factor 23 .

changed in opposite directions over time. Online supplementary Table 1 shows median FGF3 levels by trajectory group at each measurement during the 24-month period.

Table 1 shows baseline characteristics. Mean age \pm SD of the total cohort was $57 \pm 14$ years, $56 \%$ were female, $67 \%$ were black, $43 \%$ had diabetes, $78 \%$ had CVD, median (interquartile range) time on dialysis was $2(0.8-$ 4.7) years, $36 \%$ had residual kidney function, and $56 \%$ used a vitamin $\mathrm{D}$ analog at baseline. The 5 trajectory groups differed by age, sex, diabetes status, years on dialysis, HEMO study treatment randomization, and vitamin $\mathrm{D}$ analog use. Among those with baseline residual kidney function, urea clearance differed among the 5 trajectory groups. With the exception of $1,25(\mathrm{OH})_{2} \mathrm{D}$, all baseline markers of mineral metabolism differed among the trajectory groups and increased from the low stable group to the elevated stable group. Baseline inflammatory markers did not differ. In the first 24 months, there were $86(53 \%)$ hospitalizations in the low stable trajectory, $70(46 \%)$ hospitalizations in the low increasing trajectory, 115 (43\%) hospitalizations in the elevated increasing trajectory, 43 (39\%) hospitalizations in the elevated decreasing trajectory, and 106(47\%) hospitalizations in the elevated stable trajectory.

\section{Predictors of FGF23 Trajectory}

Because higher FGF23 levels are consistently associated with increased mortality [3-11], we compared the 4 lower FGF23 trajectories to the elevated stable FGF23 tra- 
Table 1. Baseline characteristics

\begin{tabular}{|c|c|c|c|c|c|c|}
\hline & $\begin{array}{l}\text { Group } 1 \text { low } \\
\text { stable } \\
(n=162)\end{array}$ & $\begin{array}{l}\text { Group } 2 \text { low } \\
\text { increasing } \\
(n=151)\end{array}$ & $\begin{array}{l}\text { Group } 3 \text { elevated } \\
\text { increasing } \\
(n=268)\end{array}$ & $\begin{array}{l}\text { Group } 4 \text { elevated } \\
\text { decreasing } \\
(n=110)\end{array}$ & $\begin{array}{l}\text { Group } 5 \text { elevated } \\
\text { stable } \\
(n=228)\end{array}$ & $\begin{array}{l}\text { Total } \\
(n=919)\end{array}$ \\
\hline Age, years & $63 \pm 13$ & $60 \pm 12$ & $56 \pm 14$ & $59 \pm 15$ & $51 \pm 14$ & $57 \pm 14$ \\
\hline Gender, female & $107(66)$ & $98(65)$ & $139(52)$ & $66(60)$ & $103(45)$ & $513(56)$ \\
\hline Black & $113(70)$ & $112(74)$ & $173(65)$ & $76(69)$ & $140(61)$ & $614(67)$ \\
\hline Current smoking & $19(12)$ & $23(15)$ & $59(22)$ & $12(11)$ & $48(21)$ & $161(18)$ \\
\hline Dialysis vintage, years & $1.3(0.6-3.1)$ & $1.6(0.6-4.1)$ & $1.8(0.8-4.9)$ & $2.3(1.0-5.2)$ & $3.2(1.4-6.7)$ & $2.0(0.8-4.7)$ \\
\hline Diabetes & $94(58)$ & $78(52)$ & $109(41)$ & $48(44)$ & $67(29)$ & $396(43)$ \\
\hline Cardiovascular disease & $130(80)$ & $115(76)$ & $217(81)$ & $82(75)$ & $161(71)$ & $705(78)$ \\
\hline Vitamin D analog use & $71(44)$ & $80(53)$ & $153(55)$ & $60(56)$ & $147(65)$ & $511(56)$ \\
\hline Arteriovenous access & $146(90)$ & $143(95)$ & $257(96)$ & $100(91)$ & $218(96)$ & $864(94)$ \\
\hline \multicolumn{7}{|l|}{ MBD lab values } \\
\hline Calcium, mg/dL & $9.1 \pm 1.0$ & $9.1 \pm 0.9$ & $9.3 \pm 0.9$ & $9.5 \pm 0.9$ & $9.5 \pm 1.0$ & $9.3 \pm 1.0$ \\
\hline Phosphorus, mg/dL & $4.8 \pm 1.6$ & $5.1 \pm 1.5$ & $5.8 \pm 1.7$ & $5.9 \pm 2.2$ & $6.6 \pm 1.9$ & $5.7 \pm 1.9$ \\
\hline $25(\mathrm{OH}) \mathrm{D}, \mathrm{ng} / \mathrm{mL}$ & $14.9(10.0-20.1)$ & $14.9(9.0-24.5)$ & $14.9(10.0-24.5)$ & $14.9(10.0-20.1)$ & $16.4(11.0-27.1)$ & $14.9(10.0-24.5)$ \\
\hline $1,25(\mathrm{OH})_{2} \mathrm{D}, \mathrm{pg} / \mathrm{mL}$ & $6.0(3.0-12.1)$ & $6.7(2.7-14.9)$ & $6.0(2.7-12.2)$ & $6.0(3.0-16.4)$ & $7.4(3.3-16.4)$ & $6.0(3.0-14.9)$ \\
\hline $\mathrm{PTH}, \mathrm{pg} / \mathrm{mL}$ & $154(63-304)$ & $188(70-395)$ & $228(96-466)$ & $123(51-289)$ & $269(125-604)$ & $194(83-437)$ \\
\hline $\mathrm{FGF} 23, \mathrm{pg} / \mathrm{mL}$ & $446(221-898)$ & $602(299-1,636)$ & $2,981(1,480-6,634)$ & $3,641(2,208-7,332)$ & $18,034(8,955-32,860)$ & $2,697(735-9,897)$ \\
\hline \multicolumn{7}{|l|}{ Inflammatory lab values } \\
\hline Albumin, g/dL & $3.8 \pm 0.3$ & $3.9 \pm 0.3$ & $3.9 \pm 0.4$ & $3.9 \pm 0.3$ & $4.0 \pm 0.3$ & $3.9 \pm 0.3$ \\
\hline $\mathrm{CRP}, \mathrm{mg} / \mathrm{L}$ & $6.0(2.5-14.9)$ & $5.5(2.2-13.5)$ & $5.0(2.2-10.0)$ & $5.0(2.2-10.0)$ & $5.5(2.5-11.0)$ & $5.0(2.5-11.0)$ \\
\hline IL-6, pg/ml & $2.7(1.5-5.5)$ & $2.5(1.0-5.0)$ & $2.7(1.3-6.7)$ & $2.7(1.3-5.5)$ & $2.7(1.5-5.5)$ & $2.7(1.3-5.5)$ \\
\hline TNF- $\alpha, p g / m l$ & $30.0(16.4-44.7)$ & $27.0(16.4-40.4)$ & $30.0(20.1-44.7)$ & $27.1(16.4-40.4)$ & $27.1(18.2-44.7)$ & $30.0(18.2-44.7)$ \\
\hline IFN- $\gamma, \mathrm{pg} / \mathrm{ml}$ & $1.3(1.0-2.2)$ & $1.5(1.1-2.0)$ & $1.5(1.1-2.2)$ & $1.6(1.0-2.2)$ & $1.5(1.1-2.2)$ & $1.5(1.1-2.2)$ \\
\hline High-Kt/V assignment & $99(61)$ & $72(48)$ & $138(51)$ & $56(51)$ & $103(45)$ & $468(51)$ \\
\hline High-flux assignment & $85(52)$ & $77(51)$ & $127(47)$ & $72(65)$ & $99(43)$ & $460(50)$ \\
\hline Residual kidney function & $67(41)$ & $57(37)$ & $103(38)$ & $35(32)$ & $69(30)$ & $331(36)$ \\
\hline Residual urea clearance, $\mathrm{mL} / \mathrm{min}$ & $0.93 \pm 1.07$ & $0.82 \pm 0.46$ & $0.64 \pm 0.41$ & $0.85 \pm 0.55$ & $0.55 \pm 0.45$ & $0.73 \pm 0.64$ \\
\hline
\end{tabular}

Data are presents as $n(\%)$, mean $\pm \mathrm{SD}$, or median (IQR).

MBD, mineral bone disorder; 25(OH)D, 25-hydroxyvitamin D; 1,25(OH) 2 D, 1,25-dihydroxyvitamin D; PTH, parathyroid hormone; FGF23, fibroblast growth factor 23; CRP, C-reactive protein; IL-6, interleukin-6; TNF- $\alpha$, tumor necrosis factor- $\alpha$; IFN- $\gamma$, interferon- $\gamma$.

jectory using multinomial logistic regression to determine significant predictors of trajectory group membership. Models were adjusted for all covariables. Table 2 shows only the variables that were significantly associated with one or more trajectories. Online supplementary Table 2 shows all the variables included in the analysis. Lower baseline serum phosphorus was significantly associated with membership in all trajectory groups compared to the elevated stable trajectory. Likewise, lower baseline serum calcium was associated with all trajectory groups compared to the elevated stable trajectory; however, the association with the elevated decreasing trajectory did not reach statistical significance. Lower IL-6, diabetes, and greater residual kidney function were associated with the low stable and low increasing trajectory groups. CVD was significantly associated with the elevated increasing group. Absence of a catheter as vascular access was associated with both the low stable and elevated decreasing FGF23 trajectories while randomization to the high-dose treatment arm was associated only with the low stable FGF23 trajectory. Randomization to the high flux treatment arm was only associated with the elevated decreas- ing trajectory. Finally, lower serum $25(\mathrm{OH}) \mathrm{D}$ was significantly associated with the elevated decreasing FGF23 trajectory, while no vitamin $\mathrm{D}$ analog use at baseline was associated with the low stable group.

\section{FGF23 Trajectories and Risk of All-Cause Mortality}

During a median follow-up of 36 (23-36) months, 341 (34\%) participants died. Table 3 shows the total number and percentage of deaths for each FGF23 trajectory. Only the low stable FGF23 trajectory demonstrated a significantly reduced hazard for all-cause mortality in the fully adjusted model compared to the elevated stable trajectory.

\section{Discussion/Conclusion}

We identified 5 distinct trajectories of serum intact FGF23 among 919 HEMO study participants. A small group of participants demonstrated a high baseline FGF23 level that decreased over 24 months. Diabetes, absence of catheter as vascular access, randomization 
Table 2. ORs* (95\% CI) for variables significantly associated with FGF23 trajectories compared to the elevated stable trajectory

\begin{tabular}{|c|c|c|c|c|c|}
\hline \multirow[t]{2}{*}{ Predictor } & \multicolumn{5}{|l|}{ FGF23 trajectory } \\
\hline & low stable & low increasing & elevated increasing & elevated decreasing & elevated stable \\
\hline Diabetes & $2.58(1.51-4.41)$ & $1.96(1.18-3.28)$ & $1.32(0.86-2.03)$ & $1.26(0.74-2.16)$ & Reference \\
\hline Cardiovascular disease & $1.17(0.65-2.11)$ & $1.12(0.64-1.94)$ & $1.77(1.11-2.82)$ & $0.96(0.54-1.70)$ & Reference \\
\hline High Kt/V & $2.53(1.54-4.17)$ & $1.33(0.83-2.13)$ & $1.41(0.96-2.07)$ & $1.54(0.94-2.52)$ & Reference \\
\hline High flux & $1.61(0.99-2.63)$ & $1.43(0.90-2.29)$ & $1.22(0.83-1.79)$ & $2.64(1.60-4.38)$ & Reference \\
\hline Catheter as vascular access & $0.20(0.07-0.56)$ & $0.43(0.14-1.23)$ & $0.86(0.34-2.19)$ & $0.33(0.12-0.92)$ & Reference \\
\hline Calcium & $0.31(0.23-0.42)$ & $0.41(0.53-0.83)$ & $0.66(0.53-0.83)$ & $0.77(0.58-1.02)$ & Reference \\
\hline Phosphorus & $0.44(0.37-0.53)$ & $0.53(0.45-0.62)$ & $0.75(0.67-0.84)$ & $0.83(0.72-0.95)$ & Reference \\
\hline IL-6 & $0.71(0.54-0.90)$ & $0.70(0.53-0.90)$ & $0.85(0.69-1.04)$ & $0.83(0.63-1.10)$ & Reference \\
\hline $25(\mathrm{OH}) \mathrm{D}$ & $0.87(0.55-1.39)$ & $0.90(0.58-1.40)$ & $0.94(0.66-1.35)$ & $0.53(0.33-0.84)$ & Reference \\
\hline Vitamin D analog use & $0.46(0.27-0.77)$ & $0.62(0.38-1.02)$ & $0.75(0.50-1.13)$ & $0.65(0.39-1.10)$ & Reference \\
\hline Residual kidney function & $2.76(1.59-4.77)$ & $2.05(1.18-3.57)$ & $1.42(0.86-2.36)$ & $1.64(0.92-2.95)$ & Reference \\
\hline
\end{tabular}

* ORs for each covariable were determined by multinomial logistic regression adjusted for all other covariables: age, sex, race, smoking, dialysis vintage, cardiovascular disease, diabetes, vitamin $\mathrm{D}$ analog use, dialysis access, calcium, phosphorus, PTH, 25(OH)D, $1,25(\mathrm{OH})_{2} \mathrm{D}$, inflammatory markers, treatment assignment, residual urea clearance. The bold values are statistically significant.

FGF23, fibroblast growth factor 23; IL-6, interleukin-6; 25(OH)D, 25-hydroxyvitamin D; PTH, parathyroid hormone; 1,25(OH)2D, 1,25-dihydroxyvitamin D.

Table 3. HRs (95\% CI) for all-cause mortality

\begin{tabular}{|c|c|c|c|c|c|}
\hline FGF23 trajectory & $\begin{array}{l}\text { Low } \\
\text { stable }\end{array}$ & $\begin{array}{l}\text { Low } \\
\text { increasing }\end{array}$ & $\begin{array}{l}\text { Elevated } \\
\text { increasing }\end{array}$ & $\begin{array}{l}\text { Elevated } \\
\text { decreasing }\end{array}$ & $\begin{array}{l}\text { Elevated } \\
\text { stable }\end{array}$ \\
\hline Deaths, $n(\%)$ & $67(38)$ & $64(43)$ & $92(34)$ & $43(39)$ & $75(33)$ \\
\hline \multicolumn{6}{|l|}{ Models and analysis, median (IQR) } \\
\hline Unadjusted & $1.04(0.75-1.45)$ & $0.97(0.69-1.36)$ & $0.93(0.68-1.26)$ & $1.21(0.83-1.77)$ & Reference \\
\hline Model 1: age, race, sex & $0.73(0.52-1.04)$ & $0.77(0.54-1.09)$ & $0.78(0.57-1.07)$ & $0.96(0.65-1.41)$ & Reference \\
\hline $\begin{array}{l}\text { Model 2: model } 1+\text { diabetes, cardiovascular disease, years on dialysis, } \\
\mathrm{Kt} / \mathrm{V} \text { and flux randomization arms, AV access, serum albumin }\end{array}$ & $0.65(0.45-0.92)$ & $0.70(0.49-1.00)$ & $0.73(0.54-1.02)$ & $0.87(0.59-1.29)$ & Reference \\
\hline $\begin{array}{l}\text { Model 3: model } 2+\text { calcium, phosphorus, } \mathrm{PTH}, 25(\mathrm{OH}) \mathrm{D}, 1,25(\mathrm{OH})_{2} \mathrm{D} \text {, } \\
\text { and inflammatory markers }\end{array}$ & $0.62(0.42-0.92)$ & $0.71(0.48-1.03)$ & $0.75(0.55-1.05)$ & $0.83(0.55-1.24)$ & Reference \\
\hline Model 4: model $3+$ baseline vitamin D analog use & $0.61(0.41-0.92)$ & $0.70(0.48-1.03)$ & $0.75(0.54-1.04)$ & $0.83(0.55-1.23)$ & Reference \\
\hline Model 5: model $4+$ residual kidney function & $0.61(0.41-0.91)$ & $0.70(0.48-1.03)$ & $0.75(0.54-1.04)$ & $0.82(0.55-1.23)$ & Reference \\
\hline
\end{tabular}

FGF23, fibroblast growth factor 23; PTH, parathyroid hormone; 25(OH)D, 25-hydroxyvitamin D; 1,25(OH)2D, 1,25-dihydroxyvitamin D; HR, hazard ratio.

to the high-dose treatment arms, low baseline serum calcium, phosphorus and IL-6, less vitamin D analog supplementation at baseline, and residual kidney function were associated with the low stable FGF23 trajectory compared to the elevated stable trajectory. Meanwhile, randomization to the high flux treatment arm, absence of a catheter as vascular access, and lower serum phosphorus and $25(\mathrm{OH}) \mathrm{D}$ were significantly associated with membership in the elevated decreasing FGF23 trajectory. Only the low stable FGF23 trajectory compared to the elevated stable trajectory was associated with a reduced hazard ratio of all-cause mortality.
As opposed to the stable or increasing FGF23 trajectories observed in pre-dialysis $\mathrm{CKD}$, [12] we observed both low and high stable trajectories, increasing trajectories, and a decreasing trajectory. A decreasing FGF23 trajectory was also observed in a subset of participants in the Evaluation of Cinacalcet $\mathrm{HCl}$ Therapy to Lower Cardiovascular Events (EVOLVE) trial [19]. In a post hoc analysis of the EVOLVE trial, more participants randomized to cinacalcet demonstrated a decrease in FGF23 levels (compared to placebo) from baseline to week 20; this decrease significantly correlated with a reduction in calcium, phosphorus, and PTH levels, which was attributed to cinacalcet treatment. Nonetheless, 28 and $15 \%$ of the 1,264 EVOLVE 
participants randomized to placebo demonstrated a $\geq 30$ and $\mathrm{a} \geq 50 \%$ reduction in FGF23 levels, respectively, over the same 20 -week period. Only $3 \%$ of the participants randomized to placebo crossed over to commercially available cinacalcet during this period. Our results extend these observations by confirming a decreasing FGF23 trajectory using 2-3 measurements of FGF23 over a 24-month period among HEMO study participants.

It is important to note that unmeasured factors and changes in baseline covariables may have influenced FGF23 trajectory. For instance, intravenous iron administration, which was not accounted for in this analysis, increases intact FGF23 among dialysis patients independent of serum calcium and phosphorus levels [20,21]. Long-term control of serum phosphorus with non-calcium based phosphate binders may result in decreases in FGF23 levels $[22,23]$, while active vitamin D stimulates FGF23 production [24]. Our study did not account for changes in serum phosphorus, type of phosphate binder, or prescription of active vitamin D; however, low baseline serum phosphorus and no baseline use of active vitamin D analogues were strongly associated with the low stable FGF23 trajectory. Furthermore, lower serum phosphorus was associated with all the FGF23 trajectories compared to the high stable FGF23 trajectory.

The elevated decreasing FGF23 trajectory was significantly associated with randomization to the high-flux treatment arm of the HEMO study. In a small study of 50 prevalent hemodialysis patients, randomized to either high-flux or low-flux hemodialysis for 1 year, intact FGF23 levels and abdominal calcification scores were significantly reduced after 1 year of treatment in the highflux arm [25]. High-flux dialysis more efficiently removes other mineral metabolism markers, which in turn reduces FGF23 levels; for example, high-flux dialysis may remove more PTH compared to low-flux dialysis [26]. Though phosphorus removal is limited by mobilization from the intra- to extracellular space, one small study suggests that significantly more phosphorus is removed with high-flux hemodialysis compared to low-flux [27]. While in many countries, low-flux hemodialysis is no longer the standard of care, our results suggest that further research is needed to explore the long-term effects of higher efficiency dialysis modalities on FGF23 and outcomes.

Only the low stable trajectory compared to the high stable trajectory was associated with a decreased hazard of all-cause mortality in the fully adjusted model. This is not surprising since it is well established that higher FGF23 levels are associated with increased mortality in ESKD [5, 7-11]. Mineral metabolism and dialysis-related factors as- sociated with a low stable trajectory were lower baseline calcium, phosphorus, and IL- 6 , as well as randomization to the high-dose HEMO study treatment arm, no vitamin $\mathrm{D}$ analog use, and greater residual kidney function. Participants in the EVOLVE Trial randomized to cinacalcet who also had a reduction in FGF23 demonstrated decreased all-cause mortality. These participants also experienced a greater reduction in calcium, phosphorus, and PTH [19]. Similarly, HEMO study participants who had a low stable FGF23 trajectory were most likely to demonstrate the lowest baseline calcium and phosphorus levels. Taken together, these results suggest that phosphorus is not the only regulator of FGF23 and that calcium may influence, or be influenced by, FGF23 as well. Additionally, they suggest that lower circulating levels of all these parameters are associated with better outcomes.

In the EVOLVE trial, $a \geq 30 \%$ reduction in FGF23 was associated with reduced mortality only among participants randomized to cinacalcet. There was no change in mortality among participants randomized to placebo with a $\geq 30 \%$ reduction in FGF23 [19]. Likewise, in our HEMO study cohort, which did not use cinacalcet, the decreasing FGF23 trajectory was not associated with lower all-cause mortality. The baseline FGF23 level in the HEMO decreasing trajectory group was the second highest of all 5 trajectory groups. While it decreased by a median of $2,743 \mathrm{pg} / \mathrm{mL}$ (25\%), the final 24-month value was still twice as high as the median value of the low stable trajectory group, which may not have been low enough to affect mortality. Another explanation for the lack of association between the decreasing trajectory in the HEMO study cohort and reduced mortality is that this group was too small to show an effect in this post hoc analysis. Further investigation is required to test whether reductions in FGF23 are only associated with decreased mortality when cinacalcet is used or whether other factors influence this association.

Our study has several strengths: a diverse patient population, long follow-up time, and numerous demographic, clinical, and laboratory variables allowing for comprehensive regression model adjustment, thus reducing confounding. Nonetheless, our study also has limitations. This was a post hoc analysis and despite adjusting for numerous covariables, residual confounding may still exist. Our analysis only focused on the association of baseline variables and FGF23 trajectories and did not account for how covariables changed over time. For example, baseline absence of venous catheter and residual kidney function, which were both associated with the low stable FGF23 trajectory, may have changed over the course of 
the study. A small sample size in each trajectory group, especially the elevated decreasing group, may limit significant associations with all-cause mortality. Certain medications influence mineral metabolism markers and may change FGF23 trajectories overtime. Moreover, anemia and mineral metabolism management, which may influence FGF23 trajectory, has changed since the HEMO study was conducted and thus may limit applicability to the current dialysis population. Another limitation is lack of information on medication use aside from baseline active vitamin D analog use. Only measures of intact FGF23 levels were available; C-terminal FGF23 measurements were not used. Finally, this analysis included only prevalent dialysis patients and participants who died within the first 24 months of the HEMO trial were excluded; therefore, a survival bias may be present and limits generalizability. Future studies of incident dialysis patients are warranted.

In conclusion, we found 5 distinct FGF23 trajectories over 24 months among HEMO study participants including one trajectory that decreased over time. Only the low stable FGF23 trajectory was associated with a lower hazard ratio for all-cause mortality. Lower baseline serum calcium, phosphorus, and IL-6, randomization to the high-dose treatment arm, baseline absence of catheter as vascular access, no baseline vitamin $\mathrm{D}$ analog use, and greater baseline residual kidney function were important predictors of low stable FGF23 trajectory group membership in this post hoc analysis of the HEMO study. Given the limitations of this analysis, the clinical interpretation of these results is uncertain and should be evaluated in other dialysis cohorts.

\section{Acknowledgments}

None.

\section{Ethics Statement}

Subjects included in the clinical study described in the manuscript gave their written informed consent. The study protocol was approved by an Institutional Review Board.

\section{Disclosure Statement}

A.J. reports receiving investigational drugs for an ongoing study from Shire.

\section{Funding Source}

This research was supported by grants from the Veterans Administration CDA 5IK2CX001030-03 (A.J.) and the NIH R01 DK094796 (M.C.). None of the funders had any role in study design; collection, analysis, and interpretation of data; writing the report; and the decision to submit the report for publication.

\section{Author Contribution}

A.J., M.C., and J.K.: research idea and study design. M.C. and A.C.: data acquisition. All authors: data analysis/interpretation; Z.Y.: statistical analysis. A.J., M.C., T.I., and J.K.: manuscript preparation. Each author contributed important intellectual content during manuscript drafting or revision and accepts accountability for the overall work by ensuring that questions pertaining to the accuracy or integrity of any portion of the work are appropriately investigated and resolved.

\section{References}

1 United States Renal Data System [Internet]. Annual Data Report [Cited Nov 2015]. Available from: http://www.usrds.org/2015/view/ v2_06.aspx. 1 United States Renal Data System [Internet]. Annual Data Report [Cited Nov 2015]. Available from: http://www.usrds. org/2015/view/v2_06.aspx.

2 Evenepoel P, Rodriguez M, Ketteler M. Laboratory abnormalities in CKD-MBD: markers, predictors, or mediators of disease? Semin Nephrol. 2014 Mar;34(2):151-63.

3 Faul C, Amaral AP, Oskouei B, Hu MC, Sloan A, Isakova T, et al. FGF23 induces left ventricular hypertrophy. J Clin Invest. 2011 Nov; 121(11):4393-408.

4 Honda $\mathrm{H}$, Michihata $\mathrm{T}$, Shishido $\mathrm{K}$, Takahashi K, Takahashi G, Hosaka N, et al.;
PLOS ONE Staff. Correction: high fibroblast growth factor 23 levels are associated with decreased ferritin levels and increased intravenous iron doses in hemodialysis patients. PLoS One. 2017 Jun;12(6): e0179139.

5 Chonchol M, Greene T, Zhang Y, Hoofnagle AN, Cheung AK. Low Vitamin D and High Fibroblast Growth Factor 23 Serum Levels Associate with Infectious and Cardiac Deaths in the HEMO Study. J Am Soc Nephrol. 2016 Jan;27(1): 227-37.

6 Hsu HJ, Wu MS. Fibroblast growth factor 23: a possible cause of left ventricular hypertrophy in hemodialysis patients. Am J Med Sci. 2009 Feb;337(2):116-22.
7 Kendrick J, Cheung AK, Kaufman JS, Greene T, Roberts WL, Smits G, et al.; HOST Investigators. FGF-23 associates with death, cardiovascular events, and initiation of chronic dialysis. J Am Soc Nephrol. 2011 Oct;22(10):1913-22.

8 Gutiérrez OM, Mannstadt M, Isakova T, Rauh-Hain JA, Tamez H, Shah A, et al. Fibroblast growth factor 23 and mortality among patients undergoing hemodialysis. N Engl J Med. 2008 Aug;359(6):584-92.

9 Isakova T, Xie H, Yang W, Xie D, Anderson AH, Scialla J, et al.; Chronic Renal Insufficiency Cohort (CRIC) Study Group. Fibroblast growth factor 23 and risks of mortality and end-stage renal disease in patients with chronic kidney disease. JAMA. 2011 Jun; 305(23):2432-9. 
10 Scialla JJ, Xie H, Rahman M, Anderson AH, Isakova T, Ojo A, et al.; Chronic Renal Insufficiency Cohort (CRIC) Study Investigators. Fibroblast growth factor-23 and cardiovascular events in CKD. J Am Soc Nephrol. 2014 Feb;25(2):349-60.

11 Wolf M, Molnar MZ, Amaral AP, Czira ME, Rudas A, Ujszaszi A, et al. Elevated fibroblast growth factor 23 is a risk factor for kidney transplant loss and mortality. J Am Soc Nephrol. 2011 May;22(5):95666.

12 Isakova T, Cai X, Lee J, Xie D, Wang X, Mehta $\mathrm{R}$, et al.; Chronic Renal Insufficiency Cohort (CRIC) Study Investigators. Longitudinal FGF23 Trajectories and Mortality in Patients with CKD. J Am Soc Nephrol. 2018 Feb; 29(2):579-90.

13 Eknoyan G, Beck GJ, Cheung AK, Daugirdas JT, Greene T, Kusek JW, et al.; Hemodialysis (HEMO) Study Group. Effect of dialysis dose and membrane flux in maintenance hemodialysis. N Engl J Med. 2002 Dec;347(25):20109.

14 El-Maouche D, Dumitrescu CE, Andreopoulou P, Gafni RI, Brillante BA, Bhattacharyya $\mathrm{N}$, et al. Stability and degradation of fibroblast growth factor 23 (FGF23): the effect of time and temperature and assay type. Osteoporos Int. 2016 Jul;27(7):2345-53.
15 Jones BL, Nagin D, Roeder K. A SAS procedure based on mixture models for estimating developmental trajectories. Sociol Methods Res. 2001;29(3):374-93.

16 Nagin DS, Odgers CL. Group-based trajectory modeling in clinical research. Annu Rev Clin Psychol. 2010;6(1):109-38.

17 Nagin DS, Odgers CL. Group-based trajectory modeling (nearly) 2 decades later. J Quant Criminol. 2010 Dec;26(4):445-53.

18 Jones BL, Nagin DS. Advances in groupbased trajectory modeling and a SAS procedure for estimating them. Sociol Methods Res. 2007 May;35(4):542-71.

19 Moe SM, Chertow GM, Parfrey PS, Kubo Y, Block GA, Correa-Rotter R, et al.; Evaluation of Cinacalcet $\mathrm{HCl}$ Therapy to Lower Cardiovascular Events (EVOLVE) Trial Investigators*. Cinacalcet, Fibroblast Growth Factor-23, and Cardiovascular Disease in Hemodialysis: The Evaluation of Cinacalcet $\mathrm{HCl}$ Therapy to Lower Cardiovascular Events (EVOLVE) Trial. Circulation. 2015 Jul; 132(1):27-39.

20 Takeda Y, Komaba H, Goto S, Fujii H, Umezu $M$, Hasegawa $H$, et al. Effect of intravenous saccharated ferric oxide on serum FGF23 and mineral metabolism in hemodialysis patients. Am J Nephrol. 2011;33(5):421-6.

21 Fukao W, Hasuike Y, Yamakawa T, Toyoda K, Aichi M, Masachika S, et al. Oral versus intravenous iron supplementation for the treatment of iron deficiency anemia in patients on maintenance hemodialysis-effect on fibroblast growth factor 23 metabolism. J Ren Nutr. 2018 Jul;28(4):270-7.
22 Lin $\mathrm{HH}$, Liou HH, Wu MS, Lin CY, Huang CC. Long-term sevelamer treatment lowers serum fibroblast growth factor 23 accompanied with increasing serum Klotho levels in chronic haemodialysis patients. Nephrology (Carlton). 2014 Nov;19(11):672-8.

23 Rodelo-Haad C, Rodríguez-Ortiz ME, Martin-Malo A, Pendon-Ruiz de Mier MV, Agüera ML, Muñoz-Castañeda JR, et al. Phosphate control in reducing FGF23 levels in hemodialysis patients. PLoS One. 2018 Aug; 13(8):e0201537.

24 Liu S, Tang W, Zhou J, Stubbs JR, Luo Q, Pi $\mathrm{M}$, et al. Fibroblast growth factor 23 is a counter-regulatory phosphaturic hormone for vitamin D. J Am Soc Nephrol. 2006 May; 17(5): 1305-15.

25 Fu X, Cui QQ, Ning JP, Fu SS, Liao XH. Highflux hemodialysis benefits hemodialysis patients be reducing serum FGF23 levels and reducing vascular calcification. Med Sci Monit. 2015 Nov;21:3467-73.

26 Makar SH, Sawires HK, Farid TM, Ali WM, Schaalan M. Effect of high-flux versus low-flux dialysis membranes on parathyroid hormone. Iran J Kidney Dis. 2010 Oct;4(4):327-32.

27 Švára F, Lopot F, Valkovský I, Pecha O. Phosphorus removal in low-flux hemodialysis, high-flux hemodialysis, and hemodiafiltration. ASAIO J. 2016 Mar-Apr;62(2):176-81. 